\title{
Hybrid intelligent watermark System
}

Fardos Adnan Abdalkader

Shahbaa I. Khaleel

Nada N. Saleem

shahbaaibrkh@uomosul.edu.iq Nada_N_S@uomosul.edu.iq

College of Computer Sciences and Mathematics University of Mosul, Iraq

Received on:16/3/2010

Accepted on:16/8/2010

\section{ABSTRACT}

As a result of the development in data transfer technology a cross multimedia and internet, it has became possible to access and copy these information in unauthentical manner. This leads to penetrate digital multimedia security problem.

In this research a hybrid method is designed to protect product from unauthentication access using watermark technique with digital images, these images represent the important part in information systems and many applications. The method indicates hiding the watermark in both spatial and frequency domains using Artificial Intelligent techniques, such as neural networks and genetic algorithms by dividing the watermark depending on the important information contents. The basic important part hides in frequency domain and the second part in spatial domain using Discreet Cosine Transform DCT and Least Significant Bit LSB.

The method efficiency is measured using Peak Signal-to-Noise Ratio PSNR and Normalized Correlation Coefficient NC, Also many attacks is used to measured the watermark robustness and feasibility.

Keyword: watermark technique, Artificial Intelligent techniques, DCT

$$
\begin{aligned}
& \text { نظام العلامة المائية المهجن ذكائيا }
\end{aligned}
$$

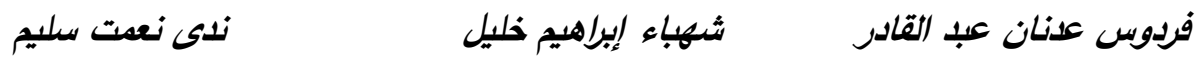

$$
\begin{aligned}
& \text { جامعة الموصل/كلية علوم الحاسوب والرياضيات }
\end{aligned}
$$

\section{الملخص}

نتيجة للتطورات الحاصلة في تقنيات نقل المعلومات والبيانات عبر الأوساط المتعددة وعلى شبكة الانترنيت أصبح بالإمكان الوصول ونسخ هذه المعلومات والبيانات بأسلوب غير مخول وهذا يودي إلى مشكلة اختراق أمنية الوسائط الرقمية.

تم في هذا البحث تصميم طريقة مهجنة لحماية المنتج من الوصول غير المخول باستخدام تقنية العلامة

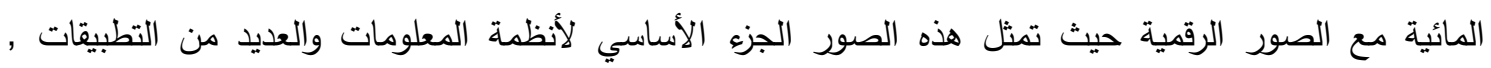
تتضمن الطريقة إخفاء العلامة المائية في المجال المكاني وفي المجال الترددي باستخدام التقنيات الذكائية التي لتي لئي تتكون من الخوارزميات الجينية والثبكات العصبية عن طريق تقسيم العلامة المائية وحسب أهمية المعلومات التي لتياهي

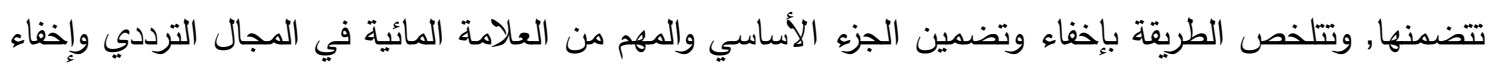

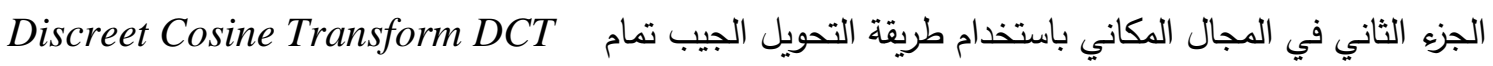
وطريقة الـ Least Significant Bit LSB 
تم قياس كفاءة الطريقة بحساب قيم الـ Peak Signal -to-Noise Ratio PSNR ومعامل الارتباط Normalized Correlation Coefficient NC

العلامة المائية. الكلمات المفتاحية:تقنية العلامة المائية, التقنيات الذكائية, المجال الترددي

1

أصبح الحاسوب الثخصي شائع جدا ومنتشر بصورة كبيرة وأصبح من السهل استتساخ وإنتاج الصور

وأفلام الفيديو والنص والصوت بسرعة وبكلفة قليلة وبالإمكان توزيع هذه الملفات بسرعة بسبب توفر الانترنت في

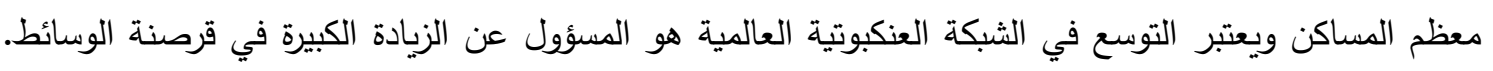

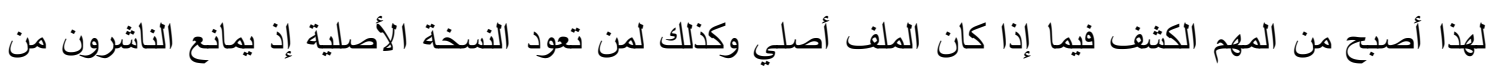

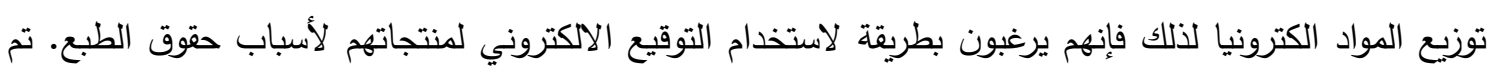

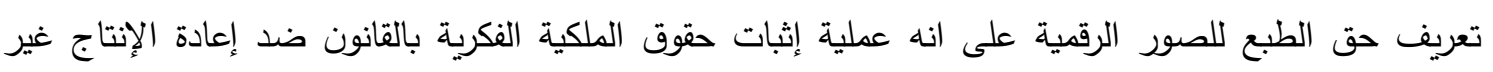
المخول به أو معالجة أو تحويل أو بث الصور الرقمية, ولتحديد المؤلف الأصلي لملف مع قوانين حقوق الطبع تم

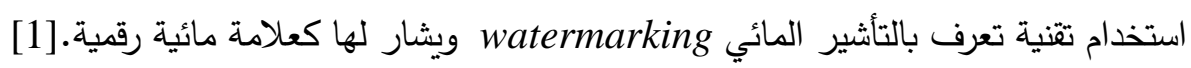

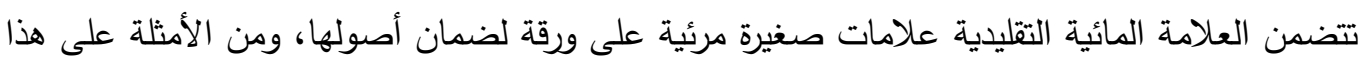
وجودها في الصكوك الحكومية والمستندات الرسمية والنقود الورقية. تم تحوير وتحديث هذه الطريقة لتلائم الصور

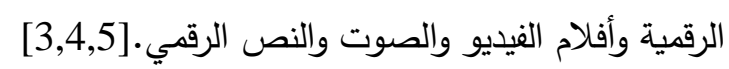

Watermark تشترك جميع طرق التأشير المائي بنفس كتل البقاء العامة. " نظام تضمين العلامة المائية embedding ونظام استرجاع العلامة المائية watermark recovery يوضح الثكل(1) العملية العامة لتصنيف العملية المائية, الإدخال إلى المخطط هو العلامة المائية، بيانات الغطاء ومفتاح اختياري سري أو عام. $[9,17$

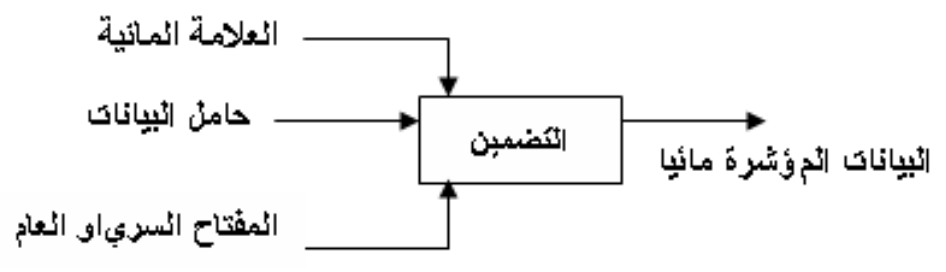

$$
\text { الثكل(1): المخطط العام للتأثير المائي الرقمي }
$$

يمكن أن تكون العلامة المائية بأي طبيعة مثل (عدد, نص, صورة) وقد يستخدم المفتاح لغرض الأمنية،

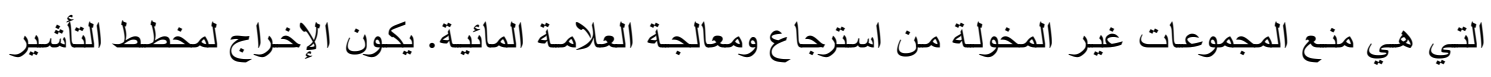
المائي هو البيانات المؤشرة مائيا. أما عملية استرجاع العلامة المائية فهو موضح بالثكل (2). نلاحظ أن الإدخال إلى المخطط هو

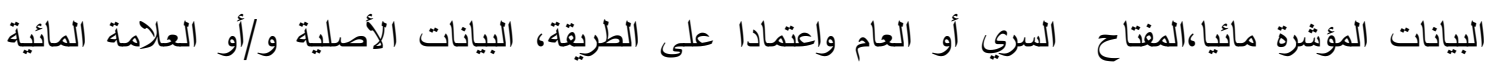

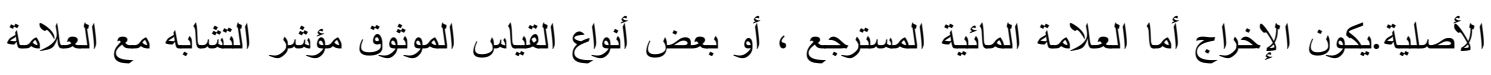
المائية في الإدخال الواجب تقديمها في البيانات. [7,8] 


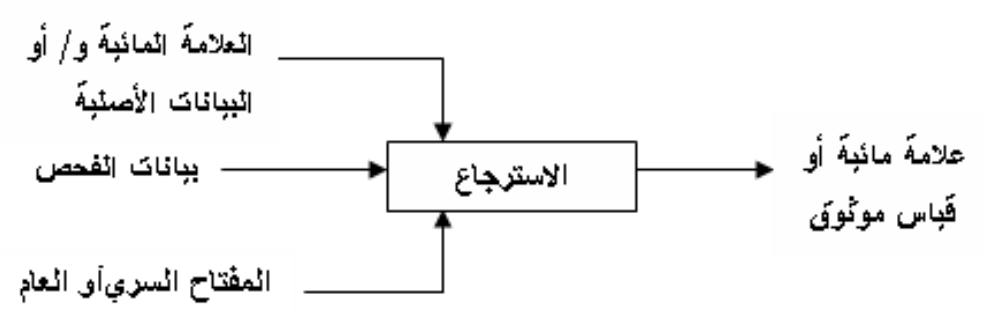

الثكل (2): المخطط العام لاسترجاع العلامة المائية

يهدف البحث إلى تصميم نظام العلامة المائية المهن للصور الرقمية. تتضمن التقنية المهنة استخدام طريقتين لإخفاء العلامة المائية, الطريقة الأولى تستخدم المجال المكاني والطريقة الثانية تستخدم المجال الترددي في الإخفاء ويتم تحديد مواقع الإخفاء باستخدام طرق التقنيات الذكائية. أن طريقة تقسيم العلامة المائية إلى جزئيين لغرض إخفائها في المجال المكاني والمجال الترددي تعتمد على المستخدم وكذلك على أهمية المعلومة التهاء التي تتضمنها العلامة المائية.

2- الاعمال السابقة

قدم الباحثون C. Osborne و A. Trikel ، R. Schyndel طريقة لتضمين العلامة المائية في المجال المكاني وهي Least Significant Bit LSB أي الخلية الاقل أهمية حيث قاموا بتضمين بيانات العلامة المائية في LSB لبعض النقاط الضوئية للصورة الأصلية (الغطاء) [18].

DCT Doomain Watermarking Techniques for Still Images: "في البحث الموسوم DCT ت Detector Performance Analysis and a New Structure حيث تم التضمين في حزمة بيانات الـ DCT ما عدا في قيمة DC وكانت عملية التضمين بجمع قيمة من قيم

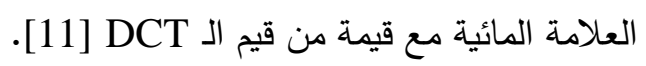

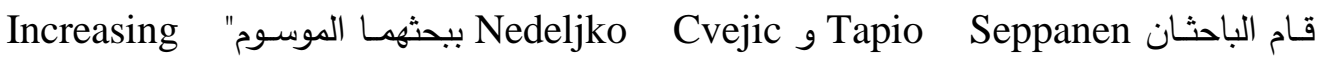
Robustness of LSB Audio Steganography Using a Novel Embedding Method طريقة جديدة وقويـة لاجراء عملية تضمين علامة مائية في ملف audio باعتماد الخانات العالية المستوى ضمن

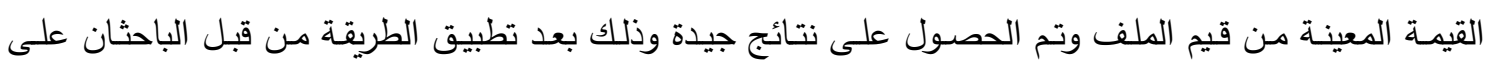
مجموعة كبيرة من انواع الموسيقى pop, rock, techno, jazz من دون حدوث تغيير مؤثر على بيانات الملف دئف

كما قدم الباحثان A. Golikeri و ط. Nasiopoulos طريقة قويـة لتضمين العلامة المائية باستخدام الـ

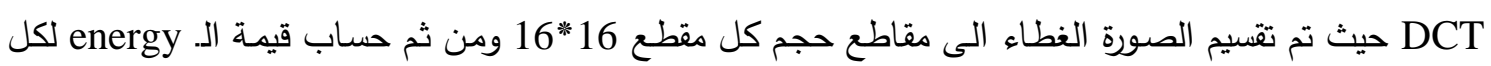

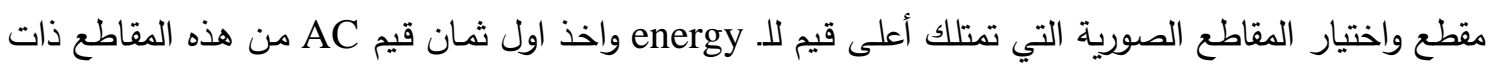
الحجم 16"16 وتضمين العلامة المائية فيها [2]. 
Robust Digital Watermarking Based Falling-off-Boundary in " قدم البحث الموسوم ضrers Board MSB6 Gray Scale Images

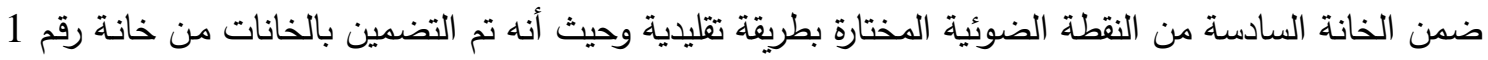

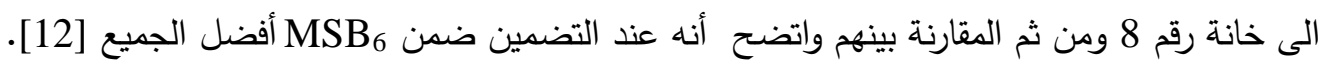

3- تقنيات الأكاء الاصطناعي

شهد العالم في الآونة الأخيرة تطوراةً كبيراًة في ميادين الحياة، ومع هذا التطور ازدادت الصعوبات التقنية

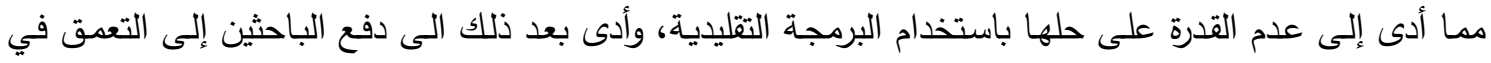

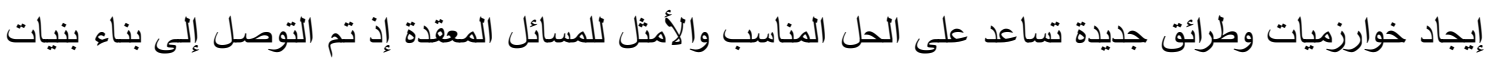

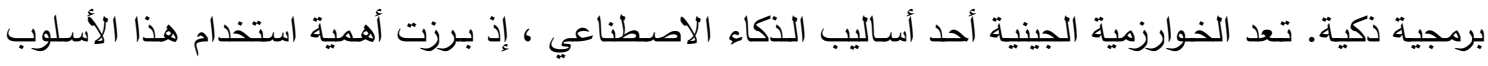

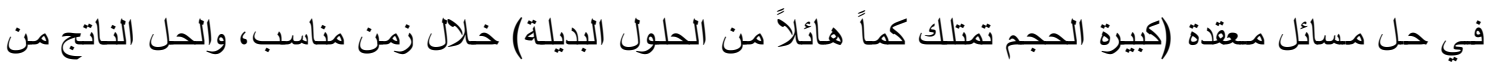

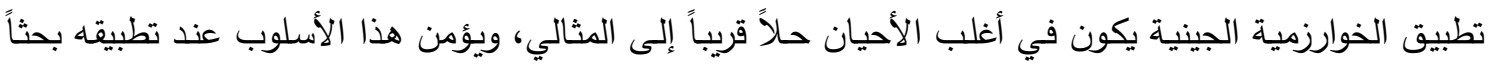

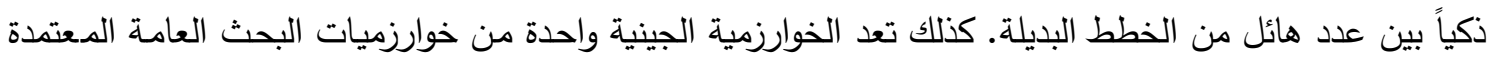

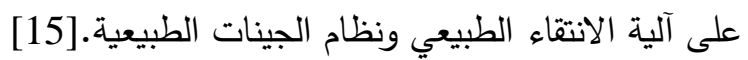
أما التقنية الأخرى من تقنيات الذكاء الاصطناعي فهي الشبكات العصبية. نشأ الاهتمام بالشبكات العصبية Neural Network من الخصائص المهمة الموجودة فيها وقدرتها على تجاوز محددات الحل التقليدي ، ومن خصائص الثبكات العصبية الاصطناعية خاصية التوازي وقدرتها على التكيف والتظظيم الذاتي ، حيث أن الشبكات العصبية توفر قدرات معالجة موثوق بها فضلا عن قابليتها على التكيف من خلال تعديل أوزان ارتباط خلاياها ، كذلك تمتلك خاصية تقسيم الذاكرة حيث إن كل خلية من خلايا الشبكة العصبية تحتوي على وحدة ذاكرة خاصة بها تقوم بخزن الحقائق ، لذا فان فقدان احد العناصر لا يسبب فقدانا لجميع البيانات المخزونة ـ تلك دلك

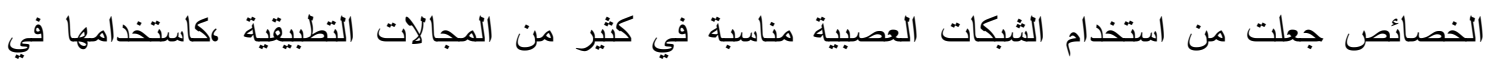
معالجة الصوت والصور الرقمية وتمييز الأنماط ـ . الخ. ولذلك كان من الضروري التوجه إلى الثبكات العصبية

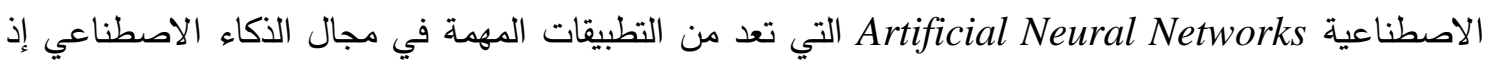
اعتمدت على أسس بيولوجية في محاولة محاكاة السلوك البشري. [19,6 تم في هذا البحث تصميم طريقة لتوليد المفتاح السري لتحديد مجموعة مواقع الغطاء byte المستخدم في تضمين واسترجاع العلامة المائية باستخدام شبكة Kohonen , وتوليد المفتاح السري لتحديد مواقع النبضات Bits في الغطاء المراد الإخفاء داخلها باستخدام الخوارزمية الجينية.

\section{4- وصف خوارزميات البحث}

\section{4-1 تضمين العلامة المائية داخل الصورة الرقمية}

إن الطريقة المهنة المستخدمة في تضمين العلامة المائية داخل الصورة الرقمية تستخدم المجالين الزماني

$$
\text { والمكاني وتتلخص بثلاث مراحل : }
$$

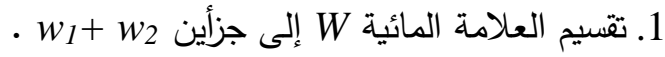


2. يتم تضمين الجزء الأول من العلامة المائية w1 في المجال المكاني Spatial domain .

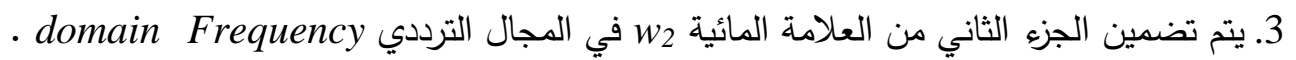

4-1-1 تقسيم العلامة المائية

إن العلامة المائية هي صورة رقمية ممثلة بالنظام الثنائي Binary Image تعتمد طريقة تقسيم العلامة

المائية على الثخص المضمن للعلامة وأهمية المعلومات والتطبيق وقد تم في هذا البحث تقسيم العلامة المائية

حسب الصورة المبينة في الثكل (3):

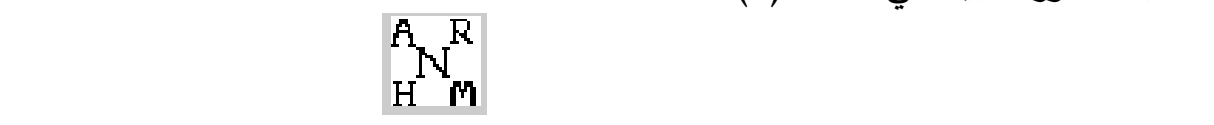

العلامة المائية الأصلية W

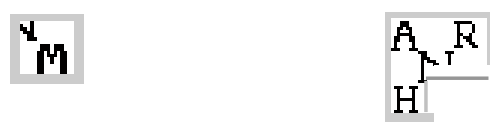

الجزء الأول من العلامة المائية الجزء الثاني من العلامة المائية

$w_{2} \quad w_{1}$

الشكل(3): تقسيم العلامة المائية

4-2 خوارزمية التهجين لتضمين العلامة المائية في الصورة الرقمية

الثكل(4) يوضح خوارزمية التضمين المهجنة .

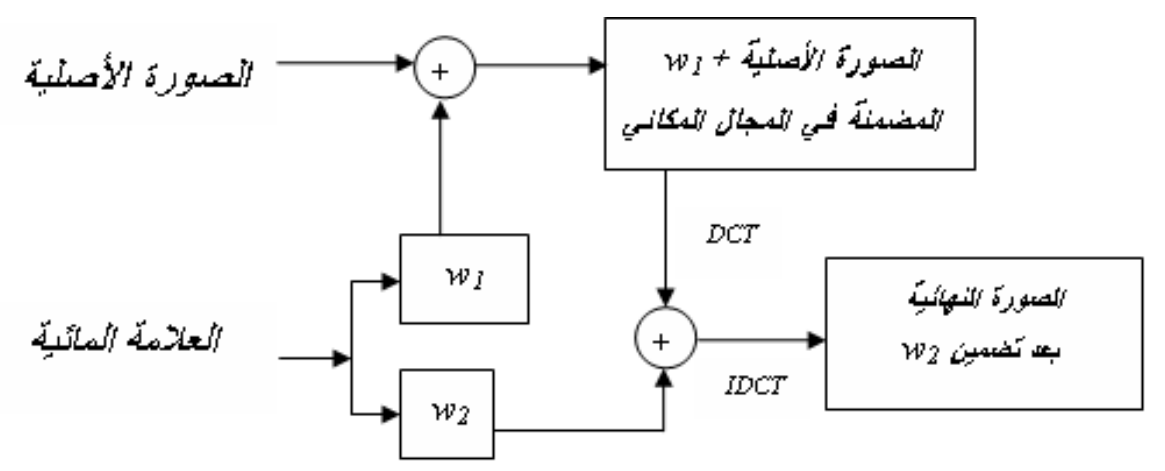

الثكل (4): خوارزمية التضمين المهن

تتلخص خوارزمية التضمين بالخطوات التالية:

1. قراءة الصورة الرقمية التي بحجم n×n وتحميلها إلى الذاكرة.

2. قراءة العلامة المائية التي بحجم m×m و وتحميلها إلى الذاكرة.

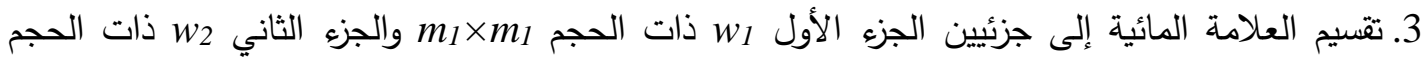

$m_{2} \times m_{2}$ 
4. توليد المفتاح السري

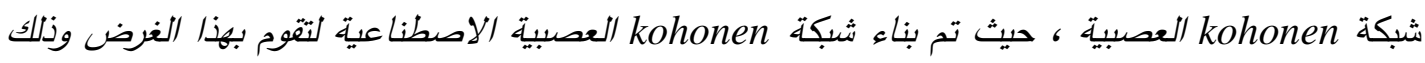
باتباع الخطوتين التاليتين.

توليد أوزان عشوائية وتتبيتها على الثبكة كأوزان إبتدائية.

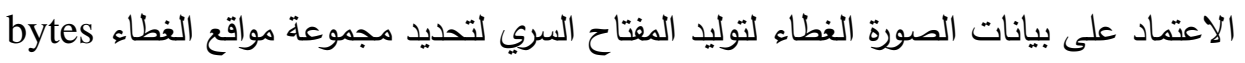

المراد الاخفاء داخلها وذلك بإدخال قيمة تلو قيمة من بيانات الغطاء الى شبكة kohonen

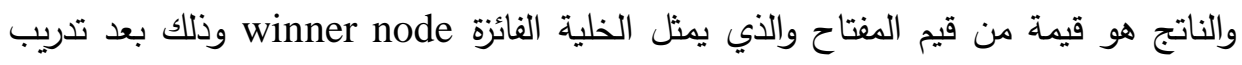
الثبكة بعدد من الخطوات ـ حيث كان عدد خلايا طبقة الاخراج هي 256 خلية وذلك لان ابعاد الصورة الغطاء كان 256*256 لكي لا ينتج موقع خارج حدود الغطاء.

5. توليد المفتاح السري الخوارزمية الجينية. بعد تحديد حجم المجتمع الممثل بحجم العلامة المائية المطلوب تضمينها ضمن الغطاء المختار تم تتفيذ الخوارزمية الجينية على جميع الافراد المحدة للحصول على المفتاح السري الذي يمثل المواقع المراد التضمين فيها أي bit وتم اعتماد التقاطع crossover ذو نقطة القطع الواحدة وبالنسبة للطفرة mutation على المفاتيح السرية لمواقع الـ bit المراد التضمين بها في المجالين المكاني والترددي بعد 100 جيل وكان أفضل حل.

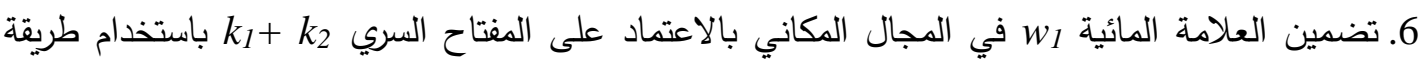
. $L S B$

7. تحويل الصورة الناتجة من عملية التضمين في المرحلة الأولى إلى المجال الترددي باستخدام تحويل جيب التمام DCT 8. يتم تضمين الجزء الثاني من العلامة المائية w2 في بيانات الصورة وهي بالمجال الترددي باستخدام المفتاح السري 9. يتم تحويل الصورة من المجال الترددي إلى المجال المكاني بعمل IDCT .

\section{4-3 خوارزمية التهجين لاسترجاع العلامة المائية المضمنة}

إن عملية استرجاع العلامة المائية من الصورة هي العملية المعاكسة لعملية التضمين حيث يكون الإدخال هنا هو الصورة المتضمنة للعلامة المائية والإخراج لهذه العملية سيكون هو العلامة المائية المسترجعة ، الثكل (5) الثينة يوضح خوارزمية الاسترجاع المهجن.

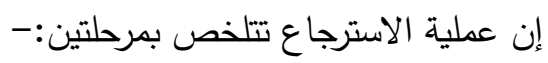

-a مرحلة استرجاع العلامة المائية من المجال الترددي للحصول على الجزء الثاني w2 من العلامة المائية. b مرحلة استرجاع العلامة المائية من المجال المكاني للحصول على الجزء الأول w w من العلامة المائية. خوارزمية استرجاع العلامة المائية تتم باستخدام الخطوات التالية:1- يتم تحويل الصورة المضمنة من المجال المكاني إلى المجال الترددي.

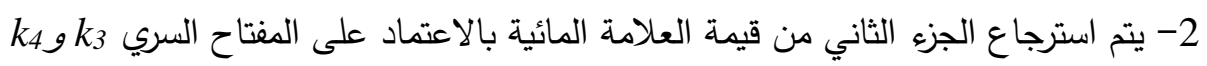




$$
\text { وخزنها في } 22
$$

3- تحويل الصورة بعد عملية الاسترجاع من المجال الترددي إلى المجال المكاني.

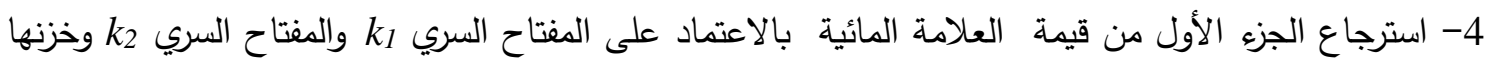
في 11 في

5- يتم دمج الجزء الأول من العلامة المائية w11 مع الجزء الثاني w22 للحصول على العلامة مائية كاملة. 6- يتم عرض العلامة المائية المسترجعة. 7- يتم حساب درجة الترابط بين العلامة المائية الأصلية والعلامة المائية الناتجة من الاسترجاع.

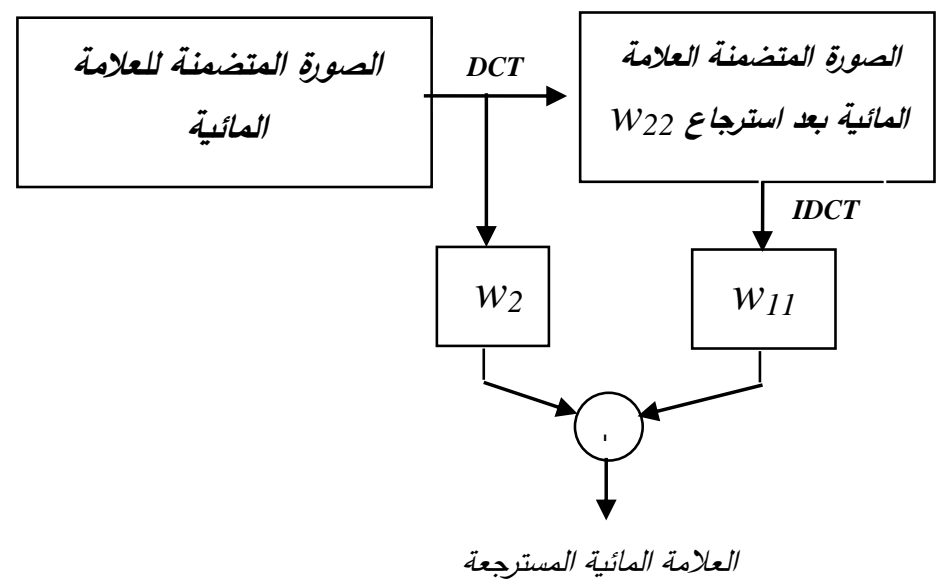

$$
\text { الثكل (5): ارزمية الاسترجاع المهن }
$$

5- -5 - حساب نسبة الخطأ والتطابق

يتم حساب نسبة الخطأ بين الصورة الأصلية والصورة المتضمنة العلامة المائية عن طريق حساب قيمة

[13,10]: باستخدام القانون التالي Peak Signal-to-Noise Ratio PSNR

$$
\begin{aligned}
& P S N R=10 \cdot \log _{10}\left(\frac{255^{2}}{M S E}\right) d B \\
& M S E=\frac{1}{n \cdot n} \sum_{i=0}^{n-1} \sum_{j=0}^{n-1}(I(i, j)-I w(i, j))^{2}
\end{aligned}
$$

حيث إن II I تمثل الصورة الأصلية و (i,j) تمثل الصورة المتضمنة العلامة المائية، كذلك ممكن حساب نسبة الخطأ بين العلامة المائية الاصلية والعلامة المسترجعة باعتماد نفس المعادلات اعلاه ، يجب أن تكون العلامة المائية المسترجعة مشابهة للعلامة المائية الأصلية ويتم حساب نسبة التطابق من خلال حساب معامل الارتباط Normalized Correlation Coefficient

$$
N C=\frac{\sum_{i=1}^{M 1} \sum_{j=1}^{M 2} w(i, j) w^{*}(i, j)}{\sqrt{\sum_{i=1}^{M 1} \sum_{j=1}^{M 2} w(i, j)^{2} \sum_{i=1}^{M 1} \sum_{j=1}^{M 2} w^{*}(i, j)^{2}}}
$$


حيث إن w(i,j) تمثل العلامة المائية الأصلية و(i,j) w تمثل العلامة المائية المسترجعة .

$$
6 \text {-6تائج عملية التضمين والاسترجاع }
$$

تم اعتماد صورة Lena ذات التدرج الرمادي القياسية بأبعاد 256*256 كغطاء للعلامة المائية. أما

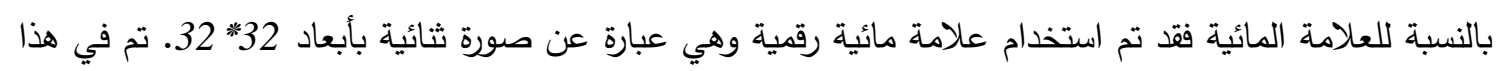

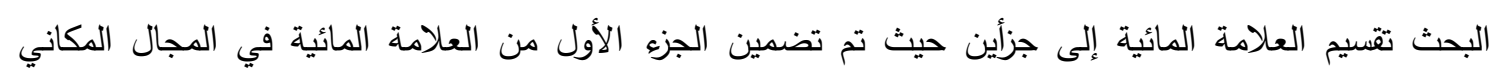

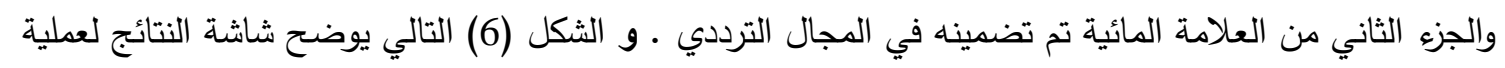
تضمين العلامة المائية بحجم 32*32 داخل الصورة Lena ذات التدرج الرمادي وبحجم 256*256.

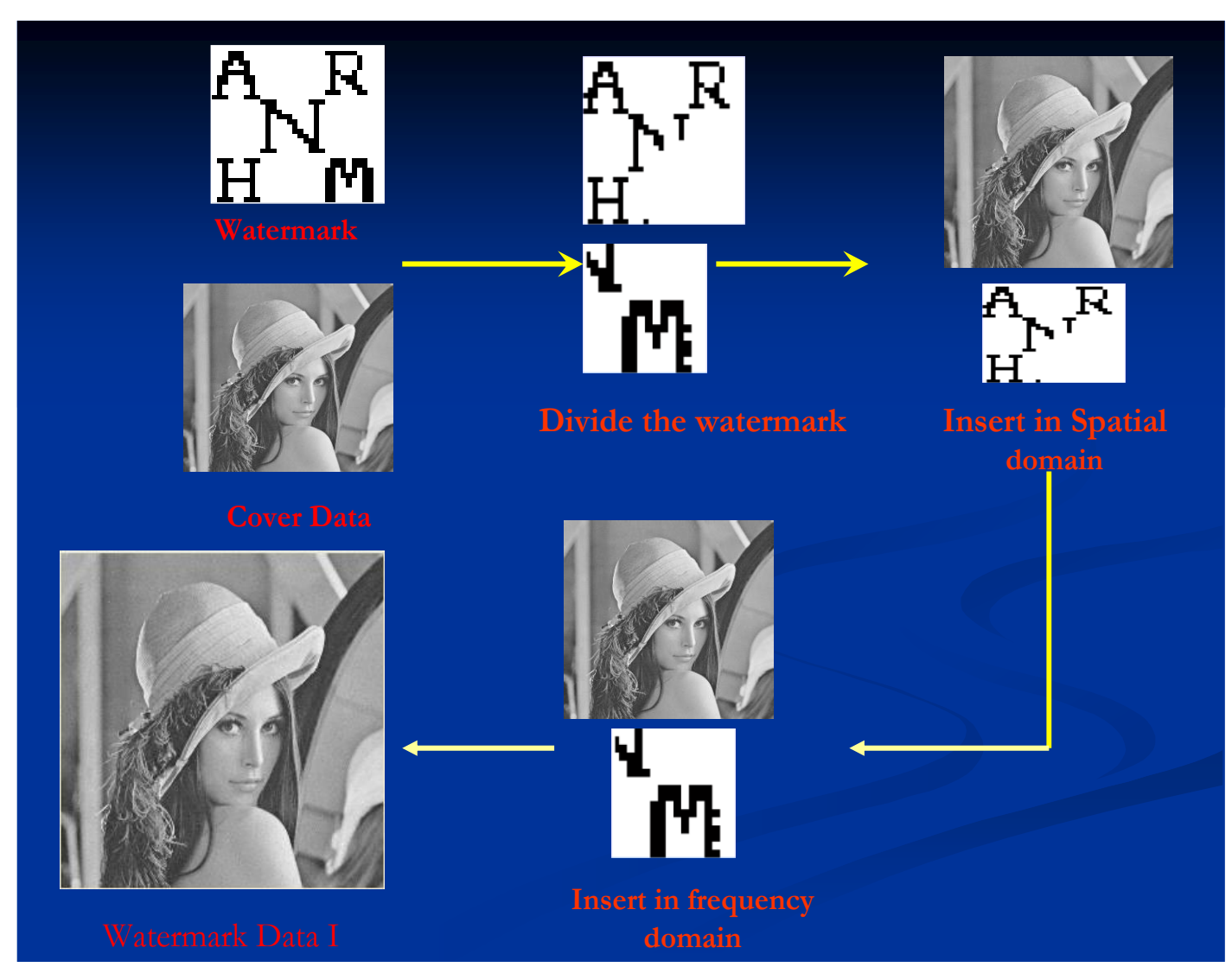

الثكل (6): يوضح شاشة النتائج لعملية تضمين للعلامة المائية

الثكل (7) التالي يوضح شاشة النتائج لعملية الاسترجاع للعلامة المائية من الصورة Lena ذات التدرج الرمادي. 


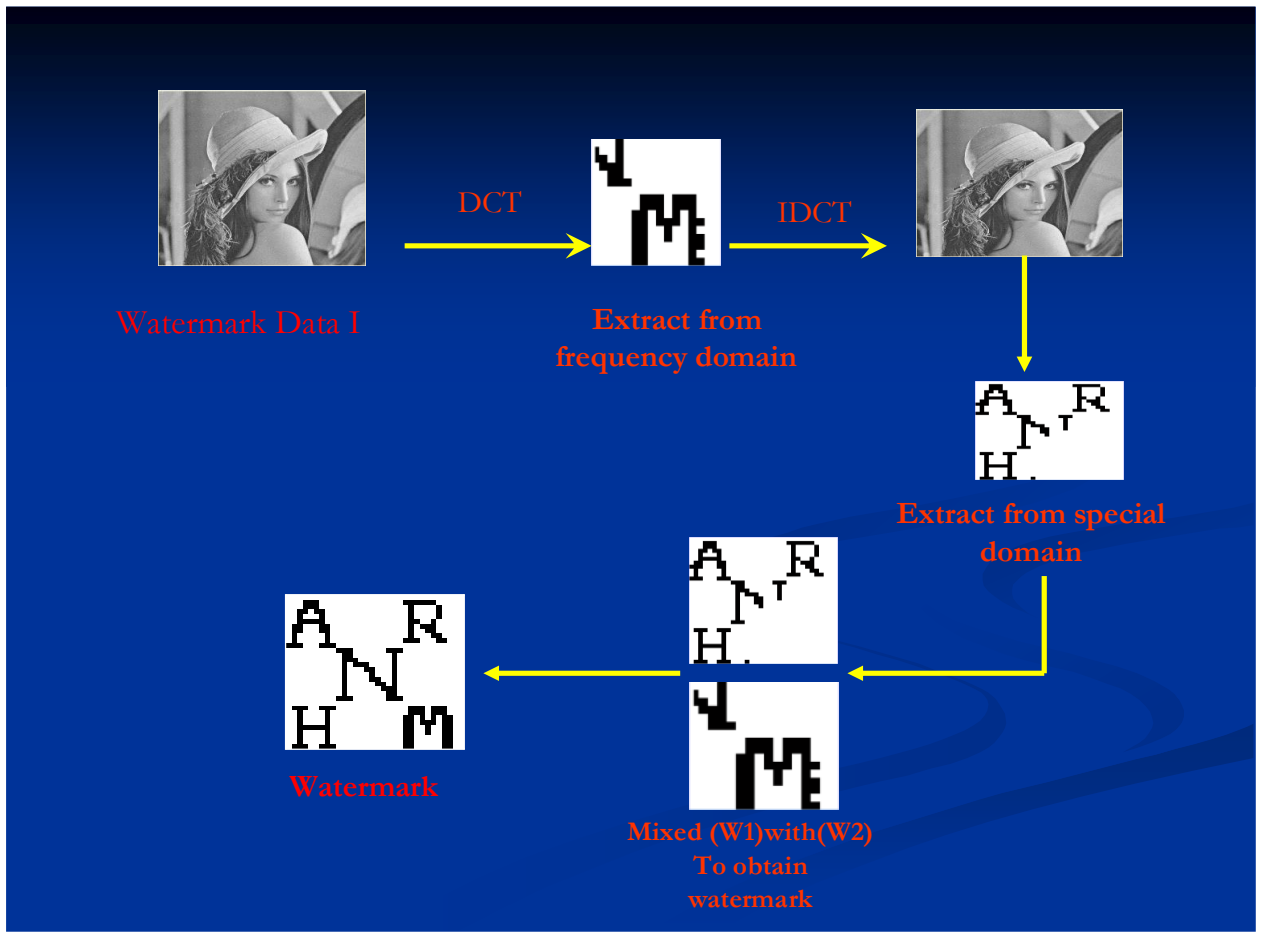

يتم حساب معامل الارتباط Normalized correlation coefficients لقياس مدى ترابط العلامـة

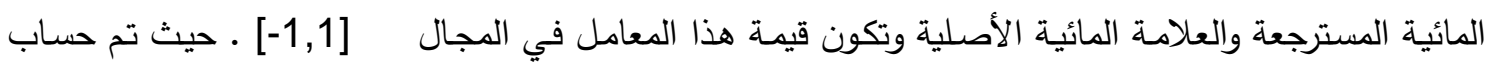

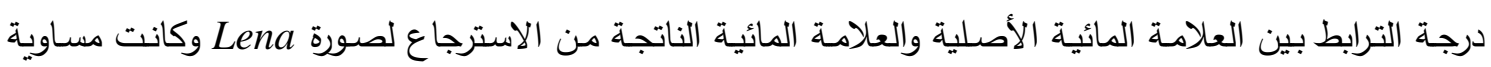

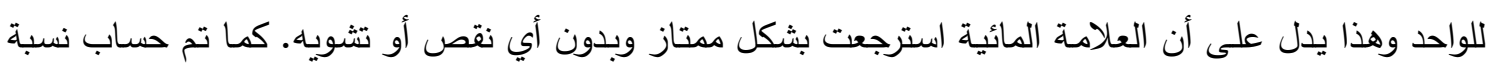
التشوه للغطاء المستخدم بعد تضمين العلامة المائية فيه عن طريق PSNR وهي اختصار لـ- Peak Signal -to Noise Ratio المستخدمة المفاتيح الذكائية.

لاختبار قوة ومتانة نظام تضمين العلامة المائية المستخدم في هذا البحث تم توجيه وإدخال بعض

التأثيرات التي تؤثر على الصورة حيث هنالك أنواع مختلفة من الهجمات في العلامات المائية والتي تعتمد على تصلى فئه

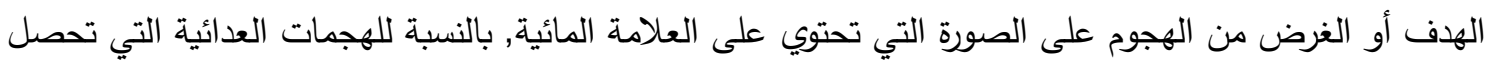
خلال أجراء عمليات معالجة الصور الثائعة التي يكون الهدف منها هو إضافة تثويه إلى العلامة المائية بالترتيب

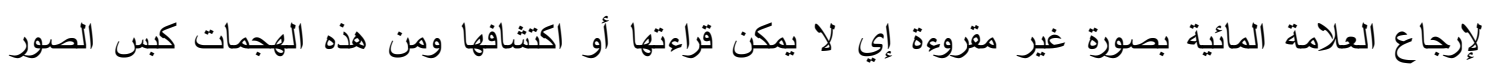

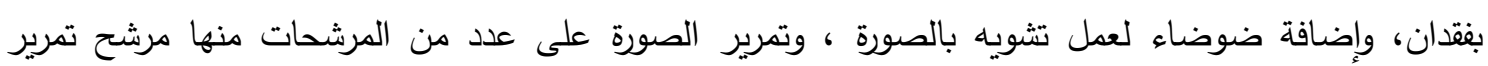

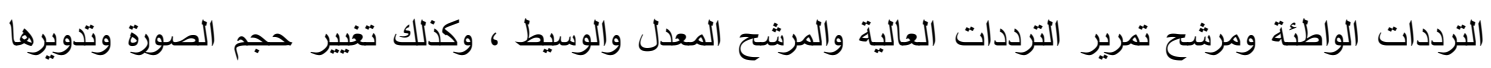

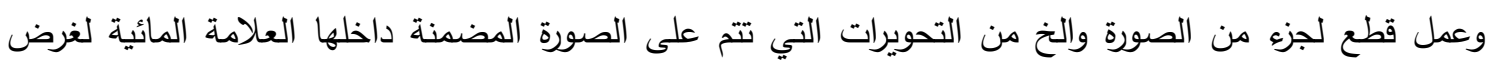
تثويهها والتي يكون القصد منها تقليل قوة العلامة المائية وعدم استرجاعها بصورتها الأصلية. تم في هذا البحث تسليط مجموعة من الهجمات على الصورة المضمنة فيها العلامة المائية مثل عمل تصغير ولئه

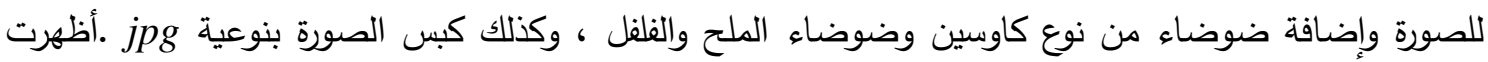


النتائج أن الخوارزمية المستخدمة في عملية التضمين والاسترجاع قوية ضد عملية الكبس، وتمرير الصورة على

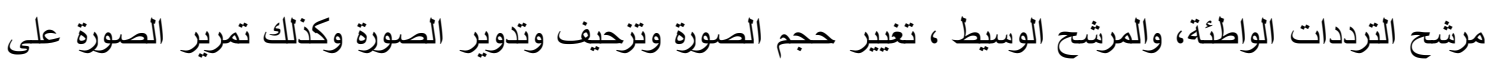
مرشح wiener والثكلان (8) و (9) يوضحان العلامة المائية المسترجعة بعد تسليط بعض التأثيرات على الصورة المضمنة للعلامة المائية بطريقتين الأولى باستخدام مفتاح سري تقليدي والأخرى باستخدام مفتاح سري ذكائي.
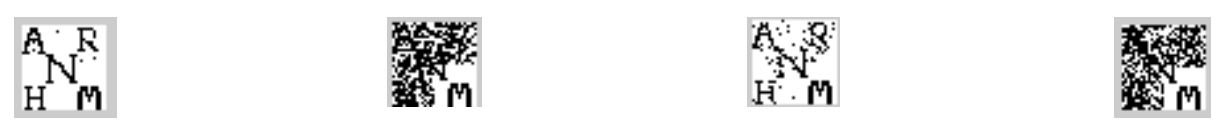

a) 0.02 salt \&pepper b) Gaussian noise c) jpeg compression

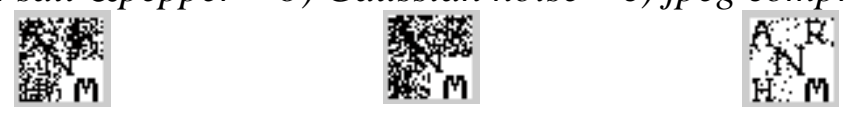

e) median filter $\quad$ f) wiener filter
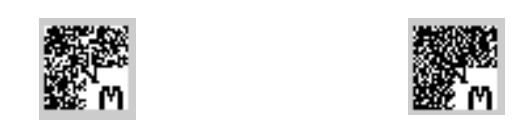

g) translate by 10

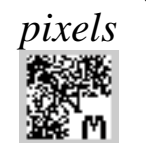

d) low pass filter

i) resizing $256-128-\quad$ j) cropping 256

k) sharpening

الثكل (8) يمثل العلامة المائية المسترجعة بعد تسليط الهجمات عليها بطريقة استخدام مفتاح سري تقليدي

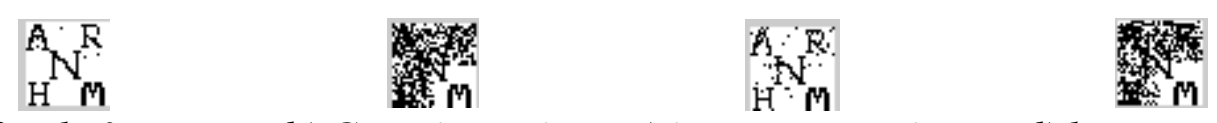

a) 0.02 salt \&pepper b) Gaussian noise

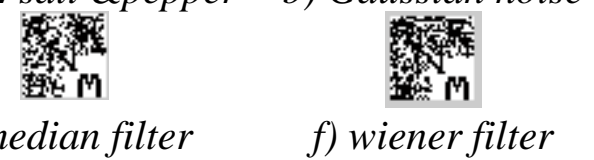

c) jpeg compression

d) low pass filter

e) median filter

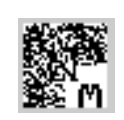

i) resizing $256-128$ -

256

الثكل (9) يمثل العلامة المائية المسترجعة بعد تسليط الهجمات عليها بطريقة استخدام مفتاح سري ذكائي

والجدولان (1) و(2) يوضحان النتائج التي تم التوصل إليها بعد تعريض الصورة المضمنة للعلامة المائية لبعض

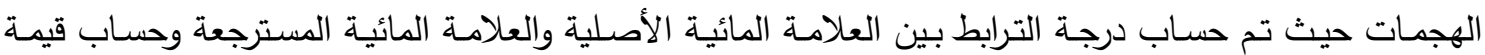


جدول(1) معامل الارتباط Normalized Correlation Coefficient ونسبة التشوه PSNR بين العلامة المائية المسترجعة والعلامة المائية الأصلية باستخدام مفتاح سري تقليدي

\begin{tabular}{|c|c|c|}
\hline Attack & $\begin{array}{c}\text { Normalized Correlation } \\
\text { Coefficient }\end{array}$ & PSNR \\
\hline Cropping & 0.710482 & 52.063800 \\
\hline Gaussian noise & 0.769484 & 52.906259 \\
\hline Jpg compression & 0.962356 & 60.309886 \\
\hline Low pass filter & 0.771037 & 52.931806 \\
\hline Median filter & 0.810369 & 53.654984 \\
\hline Resizing & 0.762456 & 52.793123 \\
\hline Salt and pepper noise & 0.996406 & 70.452291 \\
\hline Sharpening & 0.785637 & 53.182303 \\
\hline Translate & 0.967164 & 60.909866 \\
\hline Rotating & 0.883799 & 55.609292 \\
\hline Wiener filter & 0.771812 & 52.944636 \\
\hline
\end{tabular}

جدول(2) معامل الارتباط Normalized Correlation Coefficient ونسبة التثوه PSNR بين العلامة المائية المسترجعة والعلامة المائية الأصلية باستخدام مفتاح سري ذكائي

\begin{tabular}{|c|c|c|}
\hline Attack & $\begin{array}{c}\text { Normalized Correlation } \\
\text { Coefficient }\end{array}$ & PSNR \\
\hline Cropping & 0.779523 & 53.075065 \\
\hline Gaussian noise & 0.777217 & 53.035523 \\
\hline Jpg compression & 0.972208 & 61.606225 \\
\hline Low pass filter & 0.787917 & 53.223211 \\
\hline Median filter & 0.825723 & 53.984987 \\
\hline Resizing & 0.774133 & 52.983355 \\
\hline Salt and pepper noise & 0.996406 & 70.452291 \\
\hline Sharpening & 0.787158 & 53.209532 \\
\hline Translate & 0.967164 & 60.909866 \\
\hline Rotating & 0.883799 & 55.609292 \\
\hline Wiener filter & 0.792458 & 53.306199 \\
\hline
\end{tabular}

بالنسبة لقيمة معامل الارتباط كلما اقتربت من الواحد هذا يعني ان العلامـة المائية استرجعت بأفضل شكل ومن الجدولين 1 و 2 نلاحظ أنه قيم معامل الارتباط تكون أفضل عند استخدام مفتاح سري ذكائي عن ما هو عليه عند استخدام مفتاح تقليدي، حيث عند عمل cropping للصسورة المضـنة بها العلامـة المائية ومن ثم استخلصت

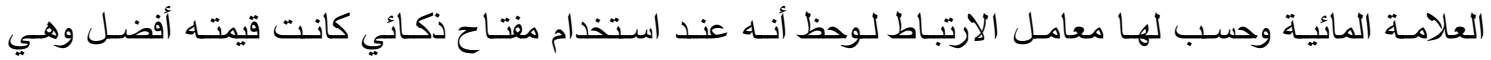

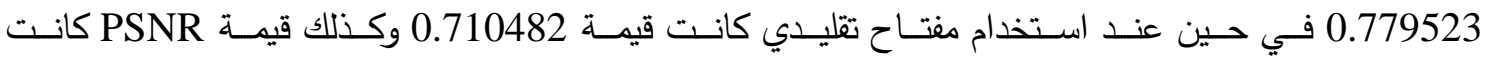
53.075065 بالذكائي (وهذا يعني أن العلامـة المائيسة المسترجعة مطابقـة للأصلية أو مشـابهة لها جداً ) بينما بالتقليدي كانت قيمته أقل وهي 52.063800 وهكذا بالنسبة لباقي الهجمات. 


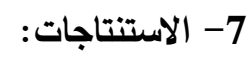

تعتبر الطريقة المعتمدة في البحث لإخفاء العلامة المائية من طرق الإخفاء القوية والجيدة إذ أنها تمكننا

استخدام العلامة المائية بحجم اكبر من الحجم المستخدم في الطرق الأخرى لأنها تستخدم الإخفاء في

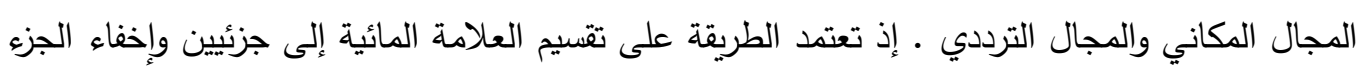

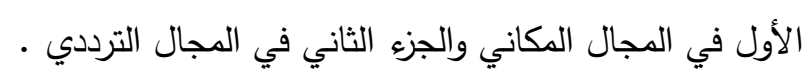

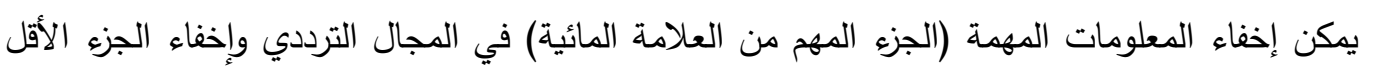

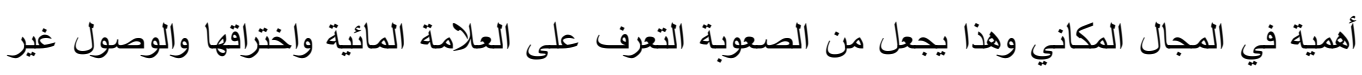
المخول لها. تميزت الخوارزميـة بالقوة والمتانـة تجاه معظم التهديدات التي تتعرض لها العلامـة المائيسة في محاولـة

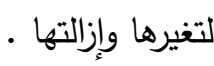
باستخدام طريقة الخوارزمية الجينية في توليد المفتاح السري تم الحصول على نتائج وقيم جيد جداً بعد الجيل المائة. كما تميزت العلامـة المائية المضمنة بكونها غير مدركة في الصورة المضمنة وتضمينها لا يسبب أي تشوه أو تغير في الصورة الحاملة لها. تم الحصول على نتائج كفؤة لقيم PSNR ومعامل الارتباط NC . 


\section{|'ll}

(2008), ، "إخفاء المعلومات الكتابة المخفية والعلامة

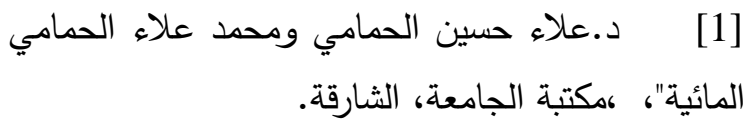

[2] A. Golikeri, P. Nasiopoulos,(2004), "A Robust DCT energy based watermarking scheme for images", Canada.

[3] AL-Hamami A.H.,Hilal M. Yousif \& Jawal M.A., (2002),"Watermarking JPEG bit Stream Images", First Conference on Information Hiding, AL-Rafidain University College Magazine, No., pp. 34-47, Baghdad, Iraq.

[4] AL-Hamami, AH \& Ziad Tariq, (2003),"An algorithm for watermarking digital images In the wavelet transform domain",second conference on information hiding, AL-Rafidian university college magazine,No. 10, Iraq.

[5] Allan M. Bruce, November $2^{\text {nd }},(2001), " A$ Review of digital watermarking", Department of Engineering, university of Aberdeen,Scotland,.

[6] Bramer M. and Devedzic V., (2004), " Artificial Intelligence Applications and Innovations, International Federation for information processing, Boston.

[7] Cox Ingemar J., Kilian Joe, Leighton Tom,and shamon Talal, (1996),"A secure, Robust watermark for multimedia", information hiding: First International workshop, proceeding, vol. 1174 of lecture notes in computer science, springer, pp. 185-205

[8] Edin Muharemagic and Borko Furht, (2004), " Multimedia security: watermarking techniques", Department of computer science and engineering, Florida Atlautic university, USA,.

[9] Fernando Perez-Gonzales and Juan R. Hernandez,(2003)," A Tutorial on digital watermarking", Dept. Of Communication vigo university , spain, project TIC96-0500-C10-10,

[10] H. Seddik, M. Sayadi, F. Fnaiech, and M. Cheriet, (2007), "New multi-domains image watermarking method based on multi-watermarks embedding and neural network segmentation, Applied mathematical sciences, vol. 1, no. 39, pp. 19271939.

[11] J. R. Hernandez , M. Amado and F. Perez-Gonzalez, " DCT Doomain Watermarking Techniques for Still Images: Detector Performance Analysis and a New Structure", in IEEE Trans. on Image processing, 9(1), pp. 55-68.

[12] M. Aliwa, T. El_Tobely, M. Fahmy, M. El Said Nasr and M. Abd El_Aziz, , August (2009), " Robust Digital Watermarking Based Falling-off-Boundary in Corners Board $\mathrm{MSB}_{6}$ Gray Scale Images", IJCSNS International Journal of Computer Science and Network Security, VOL. 9 No. 8.

[13] M. Ketcham, and S. Vongpradhip ,(2007), "Intelligent Audio Watermarking using Genetic Algorithm in DWT Domain", International Journal of Intelligent technology, vol. 2, no. 2, pp. 135-140.

[14] M. Shen, X. Zhang, L. Sun, P. Beadle and F. Chan, (2003), "A method for digital image watermarking using ICA", $4^{\text {th }}$ International symposium on 
independent component analysis and blind signal separation(ICA2003), Nara,Japan.

[15] Mitchell, M., (1996), “ An Introduction to Genetic Algorithms “, MIT press, London.

[16] N. Cvejic, T. Seppanen, (2004),"Increasing Robustness of LSB Audio Steganography Using a Novel Embedding Method", Proceedings of the International conference on information Technology: Coding and Computing, IEEE,.

[17] Nedeljno Cvejic, (2004), " Algorithms for Audio watermarking and stegano raphy", Academic dissertation, university of Oulu,.

[18] R. Schyndel, A. Trixel and C. Osborne, (1994), " A Digital Watermark", Proc. ICIP,.

[19] Zurada J. M., (1994) Introduction to Artificial Neural Systems, Jaico Publishing House, Mumbai. 